\title{
TEORES DE LIPÍDIOS TOTAIS, ÁCIDOS GRAXOS E COLESTEROL EM RESÍDUOS DESIDRATADOS DE CAMARÃO-SETE-BARBAS (Xiphopenaeus kroyeri, HELLER 1862) CAPTURADO NO ESTADO DO RIO DE JANEIRO
}

\author{
ARLAN SILVA FREITAS * \\ JOÃO TOMAZ DA SILVA BORGES ** \\ ROZENNE KERLEY COSTA *** \\ FÉLIX EMÍLIO PRADO CORNEJO **** \\ VIKTOR CHRISTIAN WILBERG ****
}

\begin{abstract}
Efetuou-se estudo sobre os lipídios totais, ácidos graxos e colesterol presentes resíduos desidratados de camarão, visando o aproveitamento total do crustáceo na alimentação humana. Resíduos de camarão-sete-barbas (cefalotórax, segmentos abdominais e caudas) foram lavados com água clorada, cozidos, desidratados, moídos e o produto final analisado. O teor de lipídios totais encontrado foi de 2,66\% e o de colesterol de $98,82 \mathrm{mg} / 100 \mathrm{~g}$. Do total dos ácidos graxos $34,43 \%$ corresponderam aos ácidos graxos saturados, $30,30 \%$ aos monoinsaturados e $35,26 \%$ aos polinsaturados. Os resíduos desidratados de camarão podem ser destinados à alimentação humana como flavorizante natural, conferindo sabor e aroma característicos ao alimento.
\end{abstract}

PALAVRAS-CHAVE: CAMARÃO-RESÍDUOS; LIPIDIOS TOTAIS; COLESTEROL; ÁCIDOS GRAXOS.

\section{INTRODUÇÃO}

Os resíduos gerados no aproveitamento do camarão (cefalotórax, segmentos abdominais e caudas) têm sido destinados à fabricação de ração animal ou simplesmente descartados.

* Químico Industrial, Mestre em Ciência e Tecnologia de Alimentos pela Universidade Federal Rural do Rio de Janeiro, (UFRRJ), RJ. (e-mail: arlanfreitas@yahoo.com.br).

** Economista Doméstico, Mestre em Ciência e Tecnologia de Alimentos, pela UFRRJ, RJ. (e-mail: jtsborges@hotmail.com.br)

*** Acadêmica, Curso de Química, Universidade Federal do Maranhão (UFMA), São Luís, MA. (e-mail: rozennecosta@bol.com.br).

**** Pesquisadores, EMBRAPA Agroindústria de Alimentos, Rio de Janeiro, RJ (e-mail: felix@ctaa.embrapa.br). 
Dados sobre a composição das frações lipídicas de subprodutos de pescados, como a farinha de camarão, são deficientes na literatura. Conforme BRAGAGNOLO e RODRIGUEZ-AMAYA (1997) os principais métodos encontrados na literatura para determinação de colesterol em camarão são colorimétricos e cromatográficos (gás). Em todos os métodos constam as etapas de extração de lipídios, saponificação, extração da matéria insaponificável e quantificação do colesterol.

CHANMUGAM et al. (1983) analisaram a composição lipídica de camarão de água doce (Macrobrachium rosenbergii) e camarão marinho (Penaeus aztecus). Constataram a predominância de ácidos graxos polinsaturados ômega seis $(\omega 6)$ nos camarões de água doce e polinsaturados ômega três ( $\omega 3)$ nos marinhos.

SHAHIDI e SYNOWIECKI (1991) relataram que os lipídios dos resíduos de camarão (Pandalus borealis) são constituídos principalmente pelos ácidos graxos insaturados.

O presente trabalho teve como objetivo determinar os teores de lipídios totais e de colesterol, bem como a composição em ácidos graxos de resíduos desidratados de camarão-sete-barbas, capturado no Estado do Rio de Janeiro, visando o aproveitamento integral do crustáceo na alimentação humana.

\section{MATERIAL E MÉTODOS}

\subsection{OBTENÇÃO DE RESÍDUOS DESIDRATADOS DE CAMARÃO}

Para obtenção de resíduos desidratados de camarão seguiu-se a metodologia descrita por FREITAS, CORNEJO e WILBERG (2001), utilizando-se os processos de cocção, secagem, moagem e adição de aditivos.

\subsection{DETERMINAÇÃO DE LIPÍDIOS TOTAIS E ÁCIDOS GRAXOS SATURADOS E INSATURADOS}

A determinação de lipídios totais nos resíduos desidratados de camarão foi realizada segundo metodologia da AOAC (1997). Para a realização da análise de ácidos graxos saturados e insaturados seguiu-se o método da AOAC (1997), utilizando cromatografia a gás (CG). As condições de 
operação do cromatógrafo foram: temperatura inicial de $100^{\circ} \mathrm{C}$, mantida durante 4 minutos, com programação de temperatura de $3{ }^{\circ} \mathrm{C} / \mathrm{min}$, atingindo a temperatura final de $240^{\circ} \mathrm{C}$ durante 15 minutos. A temperatura do injetor foi de $225^{\circ} \mathrm{C}$ e do detector de $285^{\circ} \mathrm{C}$, usando-se hélio como gás de arraste com fluxo de $0,75 \mathrm{~mL} / \mathrm{min}$. Para esta operação empregou-se coluna capilar de sílica fundida DB-WAX, com $30 \mathrm{~m}$ do comprimento e $0,30 \mathrm{~mm}$ de diâmetro interno.

\subsection{DETERMINAÇÃO DO TEOR DE COLESTEROL}

Para determinação do teor de colesterol seguiu-se o método da AOAC (1997), utilizando a Cromatografia a Gás (CG). Foram empregadas as seguintes condições de operação: coluna capilar, $1,80 \mathrm{~m} \times 2 \mathrm{~mm}$, temperaturas da coluna, injetor e detector de $245^{\circ} \mathrm{C}, 270{ }^{\circ} \mathrm{C}$ e $300^{\circ} \mathrm{C}$, respectivamente, gás de arraste (hélio) com vazão de $30 \mathrm{~mL} / \mathrm{min}$. $\mathrm{O}$ colesterol foi identificado pela comparação do tempo de retenção da amostra com o do padrão injetado. A análise quantitativa foi feita pela adição de 5- $\propto$-colestane como padrão interno e estabelecimento de curva padrão de colesterol.

\section{RESULTADOS E DISCUSSÃO}

O teor de lipídios de resíduos desidratados de camarão (2,66\%) representa cerca da metade do valor presente na carne desse crustáceo $(6,07 \%)$. KRISHNAMOORTY et al. (1979) relataram teores de 7,95\% de lipídios em camarão-rosa (Penaeus brasiliensis). Afirmaram que a contribuição dos lipídios é insignificante na composição calórica global em relação às outras fontes de energia, especialmente proteína.

No cromatograma dos ácidos graxos (Figura 1) apareceram também picos referentes a dimetilacetais (DMA).

A composição em ácidos graxos dos resíduos desidratados de camarão consta da Tabela 1. Os teores encontrados foram inferiores aos valores apresentados por CHANMUGAM et al. (1983) que analisaram camarão integral.

Os principais ácidos graxos saturados encontrados foram os ácidos palmítico (C16:0) e esteárico (C18:0), que somam 26,30\% dos lipídios totais. REDDY, NIP e TANG (1981) relataram em trabalho com camarão 
de água doce (Macrobrachium rosenbergii), que dentre os ácidos graxos saturados encontrados no crustáceo o palmítico destacou-se pela quantidade presente $(53,98 \mathrm{mg} / 100 \mathrm{~g})$.

\section{FIGURA 1 - CROMATOGRAMA DOS ÁCIDOS GRAXOS DOS RESÍDUOS DESIDRATADOS DO CAMARÃO-SETE- BARBAS OBTIDO POR CROMATOGRAFIA A GÁS}

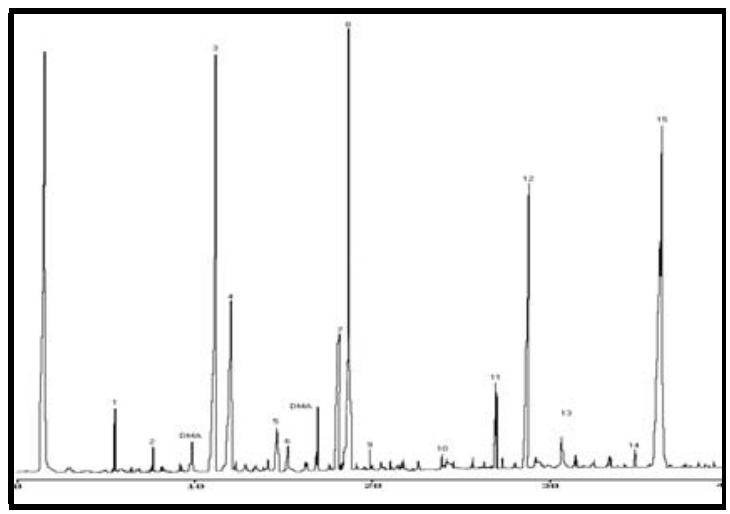

\section{TABELA 1 - DISTRIBUIÇÃO DE ÁCIDOS GRAXOS SATURADOS E INSATURADOS (g/100 g) NO RESÍDUO DESIDRATADO DE CAMARÃO}

\begin{tabular}{|c|c|c|}
\hline $\begin{array}{c}\text { cidos graxos encontrados na } \\
\text { farinhados res duos de } \\
\text { camara }\end{array}$ & $\begin{array}{l}N " \text { de carbono: } \\
N \text { "de insatura I es }\end{array}$ & $\begin{array}{l}\text { Quantidade } \\
(\mathrm{g} / 100 \mathrm{~g})\end{array}$ \\
\hline \multicolumn{3}{|l|}{ Saturados } \\
\hline $\begin{array}{l}\text { cido mir stico } \\
\text { cido pentadec lico } \\
\text { cido palm tico } \\
\text { cido marg Ærico } \\
\text { cido esteÆico } \\
\text { cido araqu dico } \\
\text { cido beh Éico }\end{array}$ & $\begin{array}{l}\text { C } 14: 0 \\
\text { C } 15: 0 \\
\text { C } 16: 0 \\
\text { C } 17: 0 \\
\text { C } 18: 0 \\
\text { C } 20: 0 \\
\text { C } 222: 0\end{array}$ & $\begin{array}{l}0,086 \\
0,038 \\
0,593 \\
0,062 \\
0,254 \\
0,025 \\
0,051\end{array}$ \\
\hline Total & & 1,109 \\
\hline \multicolumn{3}{|l|}{ Monoinsaturados } \\
\hline $\begin{array}{l}\text { cido palmitoløico } \\
\text { cido heptadecen ico } \\
\text { cido olØico } \\
\text { cidonervonico } \\
\text { Total }\end{array}$ & $\begin{array}{l}\text { C } 16: 1 \\
\text { C } 17: 1 \\
\text { C } 18: 1 \\
\text { C } 24: 1\end{array}$ & $\begin{array}{l}0,270 \\
0,038 \\
0,620 \\
0,048 \\
0,976\end{array}$ \\
\hline \multicolumn{3}{|l|}{ Polinsaturados } \\
\hline $\begin{array}{l}\text { cido linoløico } \\
\text { cido araquid nico } \\
\text { cido eicosapentaen ico (EPA) } \\
\text { cido docosapentaen ico } \\
\text { cido docosahexaen ico (DHA) }\end{array}$ & $\begin{array}{l}\text { C } 18: 2 \\
\text { C } 20: 4 \\
\text { C } 20: 5 \\
\text { C } 22: 5 \\
\text { C } 22: 6\end{array}$ & $\begin{array}{l}0,036 \\
0,157 \\
0,415 \\
0,029 \\
0,499\end{array}$ \\
\hline Total & & 1,136 \\
\hline
\end{tabular}


DENKE e GRUNDY (1991) afirmaram que a gordura rica em ácido esteárico não eleva os níveis de colesterol total em comparação com a dieta rica em ácido oléico.

Os ácidos graxos monoinsaturados que se mostraram como significativos foram os ácidos palmitoléico $(C 16: 1)$ e oléico (C18:1), os quais corresponderam a $27,62 \%$ do total. Os resultados obtidos para os ácidos graxos monoinsaturados foram superiores aos encontrados por BOTTINO et al. (1980) em camarão integral.

Os ácidos araquidônico (C20:4), eicosapentaenóico (EPA) e docosahexaenóico (DHA) destacaram-se entre os polinsaturados, correspondendo a 33,24\% dos lipídios totais encontrados nos resíduos desidratados de camarão. O EPA e DHA atuam como reguladores da ação do ácido araquidônico, que pode causar inflamação quando seus metabólitos são produzidos em excesso. Esses ácidos aumentam o "clearance" das lipoproteínas de densidade muito baixa (VLDL) do plasma. Metabolicamente, diminuem a produção hepática de triglicerídios e apolipoproteína $\mathrm{B}$, os principais constituintes lipídicos e protéicos das VLDL. Conforme MAHAN e ESCOTT-STUMPF (1998) o EPA e o DHA são precursores de prostaglandinas, tromboxanos e prostaciclinas. Grupo de componentes semelhantes ao hormônio, que participam na regulação da pressão sangüínea, freqüência cardíaca, dilatação vascular, coagulação sanguínea, lipólise, resposta imunológica e sistema nervoso central.

Os níveis de lipídios em pescados variam significativamente conforme a época, a idade, o grau de maturação sexual e a condição nutritiva, dentre outros fatores. Para OGAWA e MAIA (1999) os lipídios de animais terrestres são constituídos principalmente de ácido oléico e demais ácidos graxos saturados, enquanto que nos vegetais são representados, sobretudo pelo ácido linoléico (C18:2). Já os óleos de peixes marinhos contêm, caracteristicamente, EPA, DHA e ácido esteárico.

Quanto ao colesterol, o valor obtido para os resíduos desidratados de camarão $(98,82 \mathrm{mg} / 100 \mathrm{~g})$ foi menor que o descrito na literatura para camarão integral. KRZYNOWEK e PANUNZIO (1989) encontraram $135 \mathrm{mg} / 100 \mathrm{~g}, 139 \mathrm{mg} / 100 \mathrm{~g}$ e $161 \mathrm{mg} / 100 \mathrm{~g}$ nas espécies Pandalus borealis, Penaeus setiferous e $P$. aztecus, respectivamente. JOHNSTON et al. (1983) obtiveram valores médios de $200 \mathrm{mg} / 100 \mathrm{~g}$ de camarão. Tal discrepância pode ser atribuída à espécie do camarão, estação do ano em que foi capturado, tipo de alimentação disponível no seu habitat, tamanho e local de origem. 
Na Figura 2 é apresentado o cromatograma do colesterol dos resíduos desidratados de camarão-sete-barbas obtido por CG. Observou-se a presença de pico referente ao desmosterol, embora não se pretendesse neste trabalho determinar outros esteróis além do colesterol.

\section{FIGURA 2 - CROMATOGRAMA DO COLESTEROL PRESENTE NOS RESÍDUOS DESIDRATADOS DO CAMARÃO-SETE- BARBAS}

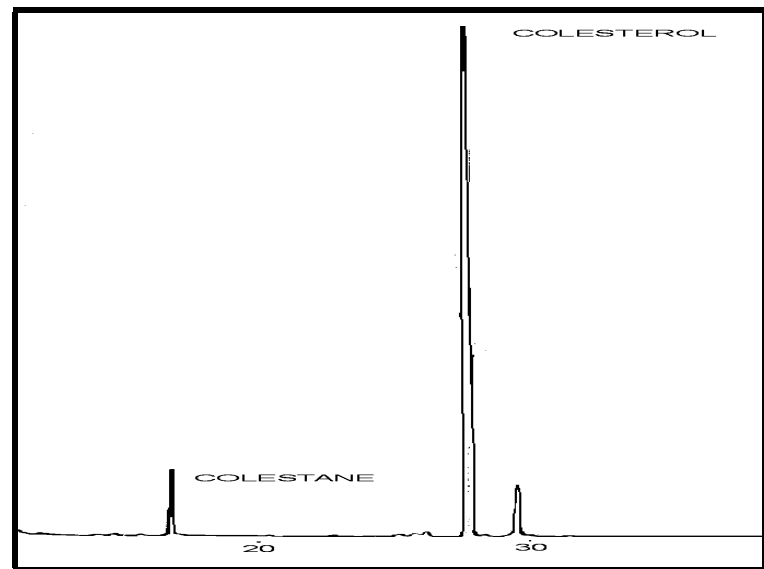

Os resíduos desidratados de camarão na forma de farinha, segundo SHAHIDI e SYNOWIECKI (1991) e FANIMO et al. (2000), apresentam baixo valor nutritivo, com enfoque protéico e consideráveis teores de cinzas $(40,00 \%)$ e fibras (12,30 g. $\left.\mathrm{kg}^{-1}\right)$. Para WANG e HWANG (2001) os sais minerais presentes na farinha dos resíduos de camarão, com destaque para o carbonato de cálcio, indicam uso potencial da mesma em dietas para fins especiais.

\section{CONCLUSÃO}

Dos ácidos graxos analisados, o mais abundante foi o ácido oléico (monoinsaturado). Dos ácidos graxos polinsaturados, os mais significativos foram os ácidos decosahexaenóico (DHA) e eicosapentaenóico (EPA), justamente os ácidos considerados de maior importância para a saúde humana. 
Os teores de lipídios totais, colesterol e ácidos graxos (saturados e insaturados) encontrados nos resíduos desidratados do camarão-setebarbas mostraram-se inferiores aos relatados na literatura para camarão integral. As características do produto indicaram que o mesmo pode ser incorporado em produtos extrusados ou em biscoitos destinados à alimentação humana.

\section{Abstract}

LEVELS OF TOTAL LIPIDS, CHOLESTEROL AND FATTY ACIDS IN THE DEHYDRATED RESIDUES OF THE SEVEN-BEARDS-SHRIMP (Xiphopenaeus kroyeri, HELLER 1862) CAPTURED IN THE STATE OF RIO DE JANEIRO

The production of shrimps in Brazil grew significantly in the last years and with excellent conditions for expansion. Although it is appreciated in the cookery, the shrimp is considered a food of high cholesterol tenor. The shrimp residues, when submitted appropriately to the cooking process, following by dehydration and reduction of particles can be destined to the human feeding as a natural flavor substance, checking to the food, flavor and aroma characteristic of shrimp. Occurred study on the total lipids, fatty acids and cholesterol, presents in total use of the crustacean in the human feeding. Residues of seven-beards-shrimp (cephalotorax, abdominal segments and tails) were washed with chlorinated water, cooked, dehydrated and grinded. In the obtained product the levels of total lipids, cholesterol and fatty acids were determinated. The total lipids and cholesterol contents were $2,66 \%$ and $98,82 \mathrm{mg} / 100 \mathrm{~g}$. From the total of the fatty acids $34,43 \%$ corresponded to the saturated, $30,30 \%$ to the monoinsatured and $35,26 \%$ to the polinsatured fatty acids.

KEY WORDS: SHRIMP-RESIDUE; TOTAL LIPIDS; CHOLESTEROL; FATTY ACIDS.

\section{REFERÊNCIAS}

1 AOAC. Association of Official Analytical Chemists. Official Methods of Analysis of AOAC International. $16^{\text {th }}$ ed. Washington, 1997. v. 2.

2 BOTTINO, N. R.; GENNITY, J.; LILLY, M. L.; SIMMONS, E.; FINNE, G. Seasonal and nutritional effects on the fatty acids of three species of shrimp, Penaeus setiferus, P. aztecus and P. duorarum. Aquaculture, v. 19, p. 139-148, 1980.

3 BRAGAGNOLO, N.; RODRIGUEZ-AMAYA, D. Otimização da determinação de colesterol por CLAE e teores de colesterol, lipídios totais e ácidos graxos em camarão rosa (Penaeus brasiliensis). Ciência e Tecnol. Alim., v. 17, n. 3, p. 275-280, 1997.

4 CHANMUGAM, P.; DONOVAM, J.; WHEELER C. J.; HWANG D. H. 
Differences in the lipid composition of fresh water prawn (Macrobrachium rosenbergii) and marine shrimp. J. Food Sci., v. 48, n. 5, p. 1440$1441,1983$.

5 DENKE, M. A.; GRUNDY, S. M. Effects of fats high in stearic acid on lipid and lipoprotein concentrations in men. Am. J. Clin. Nutr., v. 54, p. 1036-1040, 1991.

6 FANIMO, A. O.; ODUGUWA, O. O.; ONIFADE, A. O.; OLUTUNDE, T. $O$. Protein quality of shrimp waste meal. Bioresource Technology, v. 72 , n. 2, p. 185-188, 2000.

7 FReitAS, A. S.; CORNEJO, F. E. P.; WILBERG, V. C. Processo para obtenção de flavorizante natural a partir de crustáceos. Patente em publicação no INPI (Instituto Nacional de Propriedades Industriais). PI 0106361-8. Protoclo n . 000417. Data de Entrada de Reconhecimento da Patente Junto ao INPI: 11/12/2001.

8 JOHNSTON, J.J.; GHANBARI, H.A.; WHEELER, W.B.; KIRK, J.R. Characterization of shrimp lipids. J. Food Sci., v. 48, p. 33-35, 1983.

9 KRISHNAMOORTHY, R. V.; VENKATARAMIAH, A.; LAKSHMI, G.J.; BIESIOT, P. Caloric densities of shellfish meat and meat fats. J. Agric. Food Chem., v. 27, n. 5, p. 1125-1127, 1979.

10 KRZYNOWEK, J.; PANUNZIO, L. J. Cholesterol and fatty acids in several species of shrimp. J. Food Sci., v. 54, n. 2, p. 237-239, 1989.

11 MAHAN, L. K.; ESCOTT-STUMPF, S. Krause: alimentos, nutrição e dietoterapia. 9 ed. São Paulo: Roca, 1998. 1179 p.

12 OGAWA, M.; MAIA, E. L. Manual de pesca: ciência e tecnologia de pescado. São Paulo: Varela, 1999. $430 \mathrm{p}$.

13 REDDY, S. K.; NIP, W. K.; TANG, C. S. Changes in fatty acids and sensory quality of fresh water prawn (Macrobrachium rosenbergii) stored under frozen conditions. J. Food Sci., v. 46, n. 2, p. 353-356, 1981.

14 SHAHIDI, F.; SYNOWIECKI, J. Isolation and Characterization of nutrients and value-added products from snow crab (Chinoecetes opilio) and shrimp (Pandalus borealis) processing discards. J. Agric. Food Chem., v. 39, n. 8, p. 1527-1532, 1991.

15 WANG, S. L.; HWANG, J. R. Microbial reclamation of shellfish waste for the production of chitinases. Enzime and Microbial Technology, v. 28, p. 376-382, 2001. 